\title{
Inflation and long-range force from a clockwork $D$ term
}

\author{
Anjan S. Joshipura, ${ }^{*}$ Subhendra Mohanty, ${ }^{\dagger}$ and Ketan M. Patel॰ \\ Physical Research Laboratory, Navarangpura, Ahmedabad 380 009, India
}

(Received 28 November 2020; accepted 19 January 2021; published 9 February 2021)

\begin{abstract}
Cosmic inflation driven by the vacuum energy associated with the $D$ term of a supersymmetric Abelian gauge group and a possible existence of long-range force mediated by an ultralight gauge boson $Z^{\prime}$ are two extreme examples of models based on extra $U(1)$ symmetries. Large vacuum energy sets the scale of inflation while the scales of long-range forces induced by anomaly free extra gauged $U(1)$ symmetries are constrained by neutrino oscillations, binary pulsar timings, and invisible neutrino decay. There exists a difference of about 40 orders of magnitude between the scales of these two. Also, gauge couplings associated with the long-range forces are very small compared to the standard model couplings and the one required for inflation. We propose a framework based on clockwork mechanism in which these vastly different scales and associated new physics can coexist without invoking any arbitrarily small or large parameter in the fundamental theory. A chain of $U(1)$ is introduced with characteristic nearest-neighbor interactions. A large $D$ term introduced at one end governs the dynamics of inflation. $Z^{\prime}$ is localized on the other end of the chain, and it can be massless or can get naturally suppressed mass. The standard model fields can be charged under one of the intermediate $U(1) \mathrm{s}$ in the chain to give rise to their small effective coupling $g^{\prime}$ with $Z^{\prime}$. Constraints on $g^{\prime}$ and $M_{Z^{\prime}}$ are discussed in the context of the long-range forces of type $L_{\mu}-L_{\tau}, L_{e}-L_{\mu}$, and $B-L$. These, along with the inflation observables, are used to constraint the parameters of the underlying clockwork model.
\end{abstract}

DOI: 10.1103/PhysRevD.103.035008

\section{INTRODUCTION}

There exist a large number of well-motivated gauged extensions of the Standard Model (SM) containing an extra $U(1)$ group. These are proposed (i) on phenomenological grounds like explaining anomaly found in the muon anomalous magnetic moment [1] (see also [2] for a review) or as explanation of the universality violation observed in the $B$ meson decays [3], (ii) on cosmological grounds such as need to explain the dark matter [4-6], to provide secret interactions between sterile neutrinos of $\mathrm{eV}$ masses $[7,8]$ to suppress their cosmological production in the early Universe, etc., (iii) as a theoretical framework for the successful description of the inflation in the context of supersymmetric versions of the SM [9-11], and (iv) to provide a simple description of the long-range "fifth force" $[12,13]$ if it exists. Examples of such $U(1)$ are difference of any two of the leptonic charges $L_{e, \mu, \tau}$ [14-16] or an unbroken or mildly broken $B-L$ symmetry [17].

\footnotetext{
*anjan@prl.res.in

†mohanty@prl.res.in

*kmpatel@prl.res.in
}

Published by the American Physical Society under the terms of the Creative Commons Attribution 4.0 International license. Further distribution of this work must maintain attribution to the author(s) and the published article's title, journal citation, and DOI. Funded by SCOAP ${ }^{3}$.
Many extensions in category (i) and (ii) need a very light gauge boson typically in the mass range eV-MeV. The models of the $D$-term inflation [9-11] use the FayetIlliopoulos (FI) term [18] which can be written when the gauge symmetry is $U(1)$. A large value for the FI parameter $\xi \sim 10^{32} \mathrm{GeV}^{2}$ leads to inflation in the early Universe driven by an almost flat potential. Extensions in the category (iv) correspond to an entirely different parameter range. If the first generation fermions are charged under the extra $U(1)$, then the induced long-range forces are constrained by the fifth force experiments [19] or by the precision tests of gravity [20,21]. These experiments constrain the couplings of electrons to the light gauge boson $M_{Z^{\prime}}$ and are not sensitive to the neutrino couplings. Constraints on the masses and couplings for the range of length $\gtrsim 0.1 \mathrm{eV}^{-1}$ follow from these experiments and restrict the coupling $g^{\prime}$ to be $\lesssim 10^{-25}$. If $U(1)$ group distinguishes between leptonic flavors, then the long-range forces generated by electrons from the Earth, Sun, galaxies, etc., induce the matter effects in neutrino oscillations $[22,23]$. This effect can suppress the observed neutrino oscillations for a range in the gauge boson mass $M_{Z^{\prime}}$ and coupling $g^{\prime}$. Terrestrial experiments, as well as astrophysical and cosmological considerations, constrain the allowed $M_{Z^{\prime}}-g^{\prime}$ parameter space. It is found that there exists a region of parameters for which the $Z^{\prime}$ induced potential can be comparable to the Wolfenstein potential induced by the 
charged current interaction in the SM. This happens [24] for approximate ranges $M_{Z^{\prime}} \sim 10^{-17}-10^{-14} \mathrm{eV}$ and $g^{\prime} \sim$ $10^{-27}-10^{-25}$. This implies a strong hierarchy $M_{Z^{\prime}} / \sqrt{\xi} \sim$ $10^{-40}$ between the allowed ultralight mass and the inflation scale. Considering that the scales and parameters associated with the SM are much larger than $M_{Z^{\prime}}$ and $g^{\prime}$, it is natural to seek a theoretical explanation of their smallness.

It was pointed out by Fayet [25,26] (see also [27-29]) that the presence of a FI term allowed in case of the supersymmetric gauge theories can be used to relate the inflation scale $\sqrt{\xi}$ to a very small gauge coupling $g^{\prime}$. Consider a simple supersymmetric gauge theory based on a $U(1)$ group containing two oppositely charged superfields $\phi_{ \pm}$. The scalar potential of this theory includes the following $D$-term contribution:

$$
V_{D}=\frac{1}{2}\left(\xi-g^{\prime}\left(\left|\phi_{+}\right|^{2}-\left|\phi_{-}\right|^{2}\right)\right)^{2} .
$$

This simple potential is used to drive inflation when it is supplemented with a gauge singlet superfield $X$-the inflaton and an $F$ term coming from a superpotential $\lambda X \phi_{+} \phi_{-}$. A large value of the inflaton field in the early Universe leads to a supersymmetry breaking and $U(1)$ preserving minimum of $V_{D}$ with a value $\frac{1}{2} \xi^{2}$ at the minimum. For vanishing $X$ field which occurs after inflation, the $V_{D}$ has a supersymmetry preserving but the gauge symmetry breaking minimum with $g^{\prime}\left|\left\langle\phi_{+}\right\rangle\right|^{2}=\xi$. The above $D$ term leads to a scalar mass term $\mu^{2}=g^{\prime} \xi .{ }^{1}$ Requiring that this mass parameter is less than the typical supersymmetry breaking scale $\sim \mathrm{TeV}$ gives a small $g^{\prime} \leq \mathrm{TeV}^{2} / \xi \sim 10^{-26}$ $[28,29]$. Thus, a large inflation scale relates to very small value of $g^{\prime}$. While small value of $g^{\prime}$ follows in this simple $U(1)$ example, it still cannot describe the long-range forces. The $U(1)$ gauge boson in this case acquires a mass $M_{Z^{\prime}}^{2}=$ $g^{\prime 2}\left|\left\langle\phi_{+}\right\rangle\right|^{2}=g^{\prime} \xi=\mu^{2} \sim \mathrm{TeV}^{2}$ which leads to a very shortrange potential. This is an artifact of the use of the SM singlet fields $\phi_{ \pm}$for breaking the $U(1)$ symmetry. As shown by Fayet [25], it is possible to obtain ultralight or even a massless $M_{Z^{\prime}}$ [26], a small $g^{\prime}$, and the flat potential required for the inflation to start by using the SM nonsinglet fields to break the $U(1)$ symmetry. ${ }^{2}$

As an alternative to the above setup, we propose a series of $N+1$ gauged $U(1)_{i}$ groups $(i=0,1, \ldots, N)$ based on the clockwork (CW) mechanism [30,31]. $N$ chiral superfields are introduced, each of which couples to only two adjacent $U(1)$ in the chain leading to characteristic nearestneighbor interactions. FI term is introduced only for the $U(1)$ at the $N$ th site. This leads to inflation in a manner

\footnotetext{
${ }^{1}$ More precisely, this will be a $D$-term contribution to the mass of the real part of $\phi_{+}$when $g^{\prime}\left\langle\phi_{+}\right\rangle^{2}=\xi$.

${ }^{2} \mathrm{~A}$ specific example based on the $S U(5) \times U(1)$ group proposed in [25] leads to a mass relation $M_{Z^{\prime}}=\frac{g^{\prime}}{g} M_{W}$, leading to ultralight gauge boson for very small $g^{\prime}$.
}

described above. The corresponding gauge coupling is of $\mathcal{O}(1)$. All the $U(1)$ symmetries, except a linear combination of them, get broken at the minimum, but the breaking scales are hierarchically related to the FI parameter $\xi$. Specifically, the $U(1)_{i}$ is broken at a scale $\sim q^{\frac{N-i}{2}} \sqrt{\xi}$, where $q$ being the $U(1)$ charge carried by chiral superfields which induce the symmetry breaking. The remaining $U(1)$ symmetry is broken by introducing another pair of chiral superfields which couple to one of the intermediate $U(1) \mathrm{s}$ in the chain. The localization of chiral superfields away from $U(1)_{0}$ leads to an explanation of a large hierarchy between the scales of inflation and the mass of the gauge boson mediating long-range force. The SM fields also interact with one of the intermediate $U(1)$ s with $\mathcal{O}(1)$ gauge coupling. Exponentially small coupling with lightest gauge boson is then obtained in a manner used to describe the minicharged particles within the standard $\mathrm{CW}$ frameworks [31,32].

We introduce the basic framework of CW $D$ term in the next section. Inflation driven by the $D$ term along with the implications on inflationary observables is discussed in Sec. III. In Sec. IV, we collect various laboratory, astrophysical, and cosmological constraints on the popular class of long-range forces and discuss their consequences on the parameters of the $\mathrm{CW}$ framework. We summarize the study in Sec. V.

\section{FRAMEWORK}

The framework of multiple $U(1)$ we discuss here is based on the clockwork constructions discussed in [31-33]. Consider a chain of $N+1$ supersymmetric $U(1)_{i}$, with $i=0,1, \ldots, N$. A vector superfield $\mathcal{V}_{i}$ of $U(1)_{i}$ contains a vector filed $\hat{V}_{i, \mu}$, a pair of weyl fermions $\lambda_{i}, \lambda_{i}^{\dagger}$, and auxiliary field $D_{i}$. The supersymmetric Lagrangian involving gauge fields is given by

$$
\mathcal{L}_{\text {gauge }}=\sum_{i=0}^{N}\left(-\frac{1}{4} F_{i}^{\mu \nu} F_{i, \mu \nu}+i \lambda_{i}^{\dagger} \bar{\sigma}^{\mu} \partial_{\mu} \lambda_{i}+\frac{1}{2} D_{i}^{2}\right),
$$

where $F_{i, \mu \nu}=\partial_{\mu} \hat{V}_{i, \nu}-\partial_{\nu} \hat{V}_{i, \mu}$. One can further include a gauge and supersymmetry invariant FI term for each $U(1)$. Here, we assume that only $U(1)$ at the $N$ th site possesses such a term,

$$
\mathcal{L}_{\mathrm{FI}}=-\xi D_{N}
$$

The assumption of having only one vanishing FI term is technically natural [34] as the trace of each $U(1)_{i}$ factor is individually zero. We then consider $N$ pairs of chiral superfields $\Phi_{i}^{ \pm}$(with $i=1, \ldots, N$ ) charged under $U(1)_{i-1} \times$ $U(1)_{i}$ with charges $\left(\mp q_{i-1}, \pm 1\right)$. These fields are chargeless under all the other $U(1)$ in the chain. The schematic presentation of the model is displayed in Fig. 1. We also consider a chiral superfield $X$ neutral under all the $U(1)$ 


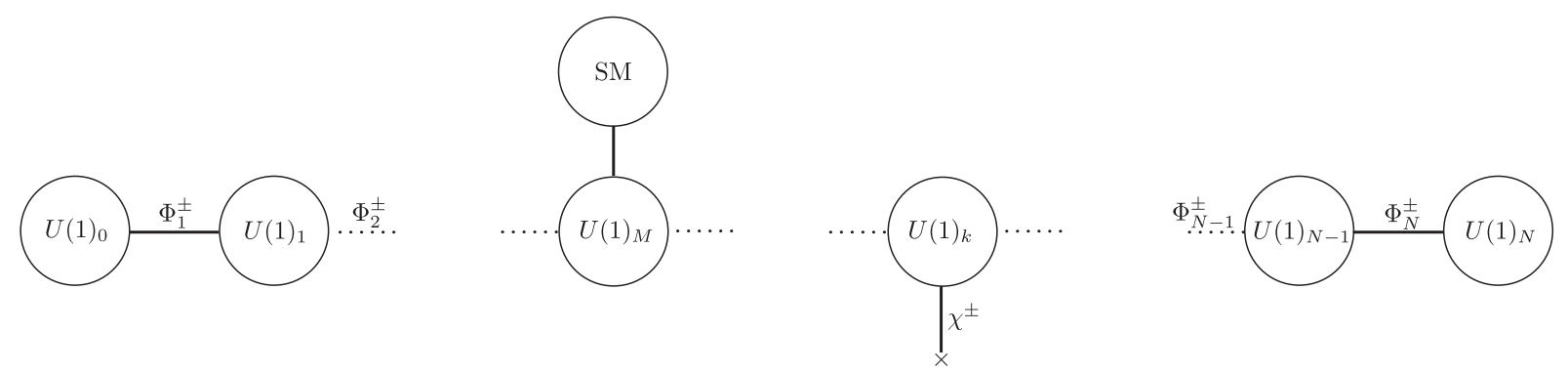

FIG. 1. Schematic presentation of the clockwork model used in the present study.

groups. The relevant superpotential considered in the underlying framework is

$$
W=\sum_{i=1}^{N} \lambda_{i} X \Phi_{i}^{+} \Phi_{i}^{-}
$$

The other terms in the potential may be forbidden by imposing additional symmetries. ${ }^{3}$ The gauge interaction between chiral and vector superfields contains the following interaction term between the scalars $\phi_{i}^{ \pm}$residing in $\Phi_{i}^{ \pm}$ and $D_{i}$ in $\mathcal{V}_{i}$ :

$$
\mathcal{L}_{\text {int }} \supset \sum_{i=1}^{N}\left(g_{i} D_{i}-g_{i-1} q_{i-1} D_{i-1}\right) G_{i},
$$

where $G_{i}=\left|\phi_{i}^{+}\right|^{2}-\left|\phi_{i}^{-}\right|^{2}$ and $g_{i}$ is the gauge coupling corresponding to $U(1)_{i}$.

Elimination of the auxiliary fields from Eqs. (2), (3), and (5) using the equations of motion implies

$$
D_{j}=\delta_{j N} \xi-g_{j}\left(G_{j}-q_{j} G_{j+1}\right)
$$

where $j=0,1, \ldots, N$ and $G_{0}=G_{N+1}=0$. Substituting this solution in Eqs. (2), (3), and (5) leads to the following $D$-term scalar potential:

$$
\begin{aligned}
V_{D}= & \frac{1}{2} \xi^{2}-\xi g_{N} G_{N} \\
& +\sum_{i=1}^{N}\left(\frac{1}{2}\left(g_{i}^{2}+g_{i-1}^{2} q_{i-1}^{2}\right) G_{i}^{2}-g_{i-1}^{2} q_{i-1} G_{i} G_{i-1}\right) .
\end{aligned}
$$

This together with the $F$-term potential derived from Eq. (4),

\footnotetext{
${ }^{3}$ For example, an $R$-symmetry under which $W \rightarrow e^{i \alpha} W$ and $X \rightarrow e^{i \alpha} X$ along with a $Z_{2}$ symmetry under which only $X$ and $\Phi_{i}^{+}$ are odd can forbid all the other terms in $W$.
}

$$
\begin{aligned}
V_{F}= & \sum_{i=1}^{N} \lambda_{i}^{2}|X|^{2}\left(\left|\phi_{i}^{+}\right|^{2}+\left|\phi_{i}^{-}\right|^{2}\right) \\
& +\sum_{i, j=1}^{N} \lambda_{i} \lambda_{j}\left(\phi_{i}^{+} \phi_{i}^{-}\right)^{*}\left(\phi_{j}^{+} \phi_{j}^{-}\right),
\end{aligned}
$$

gives the complete scalar potential of the underlying framework, $V=V_{F}+V_{D}$.

\section{A. Symmetry breaking}

It is seen from Eqs. (7) and (8) that the potential has a minimum at $\left|\phi_{i}^{ \pm}\right|=0$ when $|X|^{2} \geq g_{N} \xi / \lambda_{N}^{2}$. Consequently, the gauge symmetry is unbroken but supersymmetry gets broken by $D_{N}=\xi$. This implies an almost flat potential $V \simeq \frac{1}{2} \xi^{2}$ with nonzero slope provided by loop corrections as it will be described in Sec. III. When $X$ rolls down to its minimum, the vacuum expectation values (VEVs) of other fields are determined by the minimization of the potential $V$. The minimum of $V_{D}$ corresponds to

$G_{j}=\frac{1}{g_{j}^{2}+g_{j-1}^{2} q_{j-1}^{2}}\left(\delta_{j N} g_{N} \xi+g_{j}^{2} q_{j} G_{j+1}+g_{j-1}^{2} q_{j-1} G_{j-1}\right)$

for $j=1, \ldots, N$. These $N$ equations can be iteratively solved to find minimum for $\left|\phi_{j}^{ \pm}\right|^{2}$ along with the minimization of $V_{F}$.

For simplicity, we now assume $g_{0}=g_{1}=\ldots=g_{N-1} \equiv$ $g_{N}$ and $q_{0}=q_{1}=\ldots=q_{N-1} \equiv q$. The absolute minimum of full potential $V$ then occurs for the following:

$$
|X|=0, \quad\left|\phi_{j}^{-}\right|^{2}=0, \quad\left|\phi_{j}^{+}\right|^{2}=\frac{\mathcal{N}_{N}^{2}}{\mathcal{N}_{j-1}^{2}} q^{N-j} \frac{\xi}{g_{N}} \equiv v_{j}^{2},
$$

where

$$
\mathcal{N}_{i}^{2}=\frac{1}{1+q^{2}+q^{4}+\ldots+q^{2 i}}=\frac{1-q^{2}}{1-q^{2(i+1)}} .
$$


At this minimum, $D_{j}$ for $j=0,1, \ldots, N$ are given by

$$
D_{j}=\mathcal{N}_{N}^{2} q^{N+j} \xi
$$

Consequently, all the $D_{j}$ are nonzero and the supersymmetry is broken in each $U(1)$ sector in the true minimum. The potential $V$ at the minimum is given by

$$
V_{\text {min }}=\frac{1}{2} \mathcal{N}_{N}^{2} q^{2 N} \xi^{2} .
$$

By choosing large $N$ and $q<1$, the supersymmetry breaking effects arising from this minimum can be made small. The vacuum structure given in Eq. (10) breaks all the $U(1)$ individually but leaves a linear combination $U(1)^{\prime}$ unbroken. The corresponding generator $T^{\prime}$ can be identified in terms of $U(1)_{i}$ generators $T_{i}$ as

$$
T^{\prime}=\mathcal{N}_{N} \sum_{i=0}^{N} q^{i} T_{i}
$$

It is seen that $U(1)^{\prime}$ is dominantly localized near $U(1)_{0}$ when $q<1$.
The masses of $N+1$ gauge bosons can be obtained from the kinetic term of $\phi_{i}^{+}$using the following expression of covariant derivative:

$$
D^{\mu} \phi_{i}^{+}=\left(\partial^{\mu}+i g_{i} \hat{V}_{i}^{\mu}-i g_{i-1} q_{i-1} \hat{V}_{i-1}^{\mu}\right) \phi_{i}^{+} .
$$

We find the gauge bosons mass term

$$
\mathcal{L}_{m}=\left(\hat{M}_{V}^{2}\right)_{i j} \hat{V}_{i}^{\mu} \hat{V}_{j, \mu}
$$

where $i, j=0,1, \ldots, N$ and the elements of $(N+1) \times$ $(N+1)$ matrix are given by

$$
\left(\hat{M}_{V}^{2}\right)_{i j}= \begin{cases}g_{i}^{2}\left(v_{i}^{2}\left(1-\delta_{i 0}\right)+q_{i}^{2} v_{i+1}^{2}\right) & \text { for } j=i \\ -g_{i} g_{j} q_{j} v_{i}^{2} & \text { for } j=i-1 \\ -g_{j} g_{i} q_{i} v_{j}^{2} & \text { for } j=i+1 \\ 0 & \text { otherwise. }\end{cases}
$$

For $g_{0}=g_{1}=\ldots=g_{N-1}=g_{N}$ and $q_{0}=q_{1}=\ldots=$ $q_{N-1}=q$, the gauge boson mass matrix, at the leading order in $q$, is then given by

$$
\hat{M}_{V}^{2} \simeq \mathcal{N}_{N}^{2} g_{N} \xi\left(\begin{array}{ccccccccc}
q^{N+1} & -q^{N} & 0 & 0 & . & . & . & 0 & 0 \\
-q^{N} & q^{N-1} & -q^{N-1} & 0 & . & . & . & 0 & 0 \\
0 & -q^{N-1} & q^{N-2} & -q^{N-2} & . & . & . & 0 & 0 \\
0 & 0 & -q^{N-2} & q^{N-3} & . & . & . & 0 & 0 \\
. & . & . & . & . & . & . & . & . \\
. & . & . & . & . & . & . & . & . \\
. & . & . & . & . & . & . & . & . \\
0 & 0 & 0 & 0 & . & . & . & q & -q \\
0 & 0 & 0 & 0 & . & . & . & -q & 1
\end{array}\right) .
$$

This matrix admits a massless state which is a specific linear combination of all the $\hat{V}_{i}^{\mu}$ states given by

$$
V_{0}^{\mu} \equiv \mathcal{N}_{N} \sum_{i=0}^{N} q^{i} \hat{V}_{i}^{\mu}
$$

The other mass eigenstates $V_{j}^{\mu}$ can be determined by an approximate diagonalization of Eq. (18). Defining an orthogonal transformation

$$
\hat{V}_{i}^{\mu}=\sum_{j=0}^{N} \mathcal{R}_{i j} V_{j}^{\mu},
$$

such that $\mathcal{R}^{T} \hat{M}_{V}^{2} \mathcal{R} \equiv \operatorname{Diag} .\left(0, M_{V_{1}}^{2}, \ldots . M_{V_{N}}^{2}\right)$, we find the following form of $\mathcal{R}$ at the leading order:

$$
\mathcal{R} \approx\left(\begin{array}{ccccccccc}
1-\frac{q^{2}}{2} & -q & 0 & 0 & . & . & . & 0 & 0 \\
q & 1-q^{2} & -q & 0 & . & . & . & 0 & 0 \\
q^{2} & q & 1-q^{2} & -q & . & . & . & 0 & 0 \\
q^{3} & q^{2} & q & 1-q^{2} & . & . & 0 & 0 \\
. & . & . & . & . & . & . & . & . \\
. & . & . & . & . & . & . & . \\
\cdot & . & . & . & . & . & . & . \\
q^{N-1} & q^{N-2} & q^{N-3} & q^{N-4} & . & . & 1-q^{2} & -q \\
q^{N} & q^{N-1} & q^{N-2} & q^{N-3} & . & . & q & 1-\frac{q^{2}}{2}
\end{array}\right) .
$$

The zeros in $\mathcal{R}$ denote analytically approximated values. Numerically, for exact $\mathcal{R}$, we find their magnitudes nonzero 
but more suppressed than the other elements present in the corresponding row. The masses of physical states, $V_{j}^{\mu}$ with $j=1, \ldots, N$, are obtained as

$$
M_{V_{j}} \simeq q^{(N-j) / 2} \sqrt{g_{N} \xi}
$$

at the leading order.

A linear combination of $\hat{V}_{j, \mu}$, identified as $V_{0, \mu} \equiv Z_{\mu}^{\prime}$, is massless as a consequence of unbroken $U(1)^{\prime}$. A small mass for $Z^{\prime}$ can be generated by introducing an additional pair of chiral superfields $\chi^{ \pm}$. Let us assume that such a pair is charged under some $U(1)_{k}$ in the chain and neutral under the rest of $U(1)$. The VEVs of the scalar components of $\chi^{ \pm}$ then give an additional contribution to the gauge boson mass term Eq. (16) given by

$$
\delta \mathcal{L}_{m}=g_{k}^{2} v_{\chi}^{2} \hat{V}_{k}^{\mu} \hat{V}_{k, \mu},
$$

where $v_{\chi}^{2}=\left(\left\langle\chi^{+}\right\rangle^{2}+\left\langle\chi^{-}\right\rangle^{2}\right)$. For $v_{\chi}^{2}<v_{k}^{2}$, the leading order diagonalization of the modified gauge boson mass matrix is still given by the orthogonal matrix $\mathcal{R}$ given in Eq. (21). Using this, one obtains in the physical basis

$$
\delta \mathcal{L}_{m} \simeq g_{N}^{2} v_{\chi}^{2} \sum_{j=0}^{k+1} q^{2|k-j|} V_{j}^{\mu} V_{j, \mu} .
$$

As a result, mass of the $j$ th gauge boson gets shifted by $\delta M_{V_{j}} \simeq g_{N} v_{\chi} q^{|k-j|}$ for $j \leq k+1$. This shift gives mass to the $Z^{\prime}$ given by

$$
M_{Z^{\prime}} \simeq g_{N} q^{k} v_{\chi}
$$

Evidently, an ultralight $Z^{\prime}$ can be obtained by either localizing $\chi$ near $U(1)_{0}$ and choosing tiny $v_{\chi}$ or taking $k$ close to $N$ and appropriate $v_{\chi}$ respecting the constraint $v_{\chi} \lesssim v_{k}$.

\section{B. Coupling with the Standard Model fields}

Assume that some of the SM fields are charged under $U(1)_{M}$ in the CW chain such that $0<M \leq N$ (see Fig. 1). The coupling between the $U(1)_{M}$ current $J^{\mu}$ of these fields and the physical gauge boson of $U(1)_{j}$ is then given by a neutral current interaction term,

$$
\mathcal{L}_{\mathrm{NC}}=g J^{\mu} \hat{V}_{M, \mu} \simeq g J^{\mu} \sum_{j=0}^{M+1} q^{|M-j|} V_{j, \mu},
$$

where $g \equiv g_{M}$ and the second equality follows from Eqs. (20) and (21). For $j>M+1$, one finds the coefficient much smaller than $q^{|M-j|}$ and therefore we neglect these terms and truncate the sum at $j=M+1$. The effective coupling of $Z^{\prime}$ boson with the $J_{\mu}$ is then given by

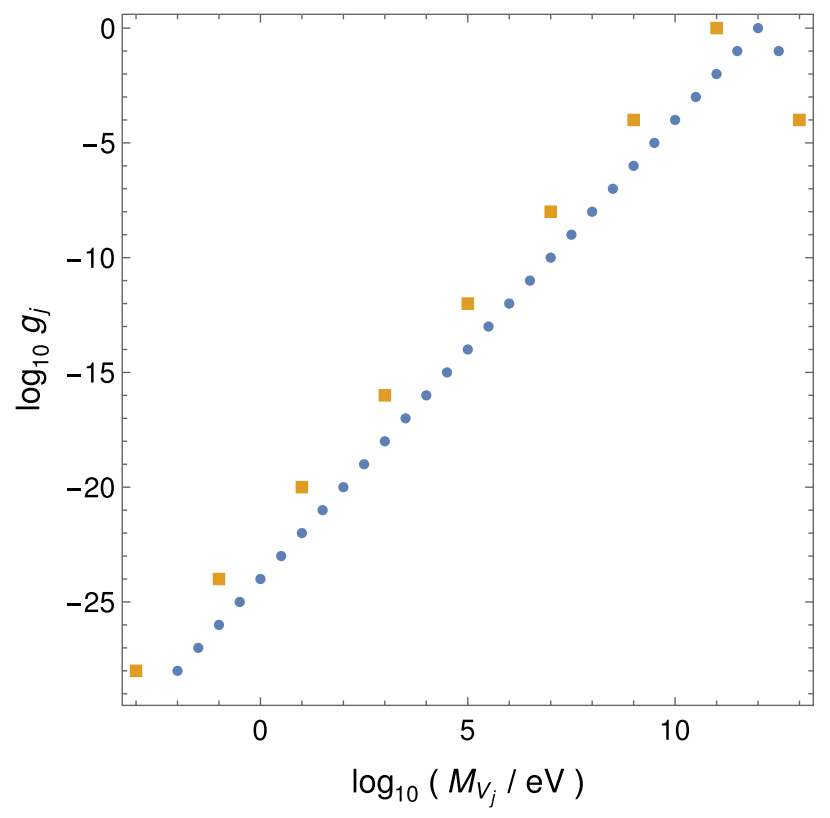

FIG. 2. Mass $M_{V_{j}}$ and coupling $g_{j}$ of the gauge boson mode $V_{j}$ $(j=1,2, \ldots, M+1)$ as obtained from Eqs. (22) and (26) for $g=1$ and $\sqrt{g_{N} \xi}=10^{16} \mathrm{GeV}$. The blue dots (orange squares) are for $N=55, q=0.1\left(N=15, q=10^{-4}\right)$, and the corresponding value of $M$ determined from Eq. (28) is 29 (8) for $M_{V_{M}}=1 \mathrm{TeV}$.

$$
g^{\prime}=g q^{M}
$$

Hence, $g^{\prime}$ can be made exponentially suppressed choosing appropriately large $M$ and $q<1$.

As seen from Eq. (26), the gauge boson with the strongest coupling to $J_{\mu}$ is $V_{M}$. If $g \sim \mathcal{O}(1)$, the mass $\sim q^{\frac{N-M}{2}} \sqrt{g_{N} \xi}$ of this gauge boson is dominantly constrained from the direct search experiments depending on the exact nature of $J^{\mu}$. Therefore, $M$ can be determined from Eq. (22) as

$$
M=N-2 \frac{\ln \left(M_{V_{M}} / \sqrt{g_{N} \xi}\right)}{\ln q},
$$

for a given $M_{V_{M}}$. Fixing $M$ in this way using a generic value $M_{V_{M}}=1 \mathrm{TeV}$, we show the couplings and masses of the gauge boson corresponding to $j=1,2, \ldots, M+1$ for two sample values of $N$ and $q$ in Fig. 2. It can be seen that one obtains $M_{V_{1}} \geq \mathcal{O}\left(10^{-3}\right) \mathrm{eV}$ for $g_{1} \geq 10^{-30}$ almost independent of the values of $N$ and $q$.

Nonobservation of supersymmetric particles in the experimental searches so far typically suggests that the supersymmetry must be broken at scale $\gtrsim$ few $\mathrm{TeV}$, at least in the visible sector. This can be achieved by introducing the usual soft terms in this framework. The minimal supersymmetric standard model fields charged under the $U(1)_{M}$ also receive supersymmetry breaking contribution from the nonvanishing $D_{j}$. However, such contribution is 
power suppressed and negligible in comparison to the soft breaking scale. For example, one obtains the largest $D_{j}$ corresponding to $j=0$ from Eq. (12), $D_{0} \simeq 10^{-5} \mathrm{eV}^{2}$ for $N=55, q=0.1$, and $\sqrt{\xi}=10^{16} \mathrm{GeV}$. Such a small contribution is insignificant for the $\mathrm{TeV}$ scale soft masses.

\section{INFLATION}

Inflation can proceed in a way analogous to the standard $D$-term inflation mechanism proposed in [9-11] (see also [35-37] for the recent versions). We identify the radial component $\sigma$ of

$$
X=\frac{1}{\sqrt{2}} \sigma e^{i \theta}
$$

as the inflaton. As discussed earlier, for the inflaton field value $\sigma^{2} \geq 2 g_{N} \xi / \lambda_{N}^{2}$, the potential has minimum at $\left|\phi_{i}^{ \pm}\right|=$ 0 and it is given by

$$
V=\frac{1}{2} \xi^{2}
$$

Nonzero $D$ term for the $N$ th $U(1), D_{N}=\xi$, spontaneously breaks supersymmetry and splits the masses of fermions and bosons residing within $\Phi_{N}^{ \pm}$. The fermion masses are given by $m_{f}=\lambda_{N}|X|$ while the masses of scalars, as can be read off from Eqs. (7) and (8), are given by $m_{ \pm}^{2}=\lambda_{N}^{2}|X|^{2} \mp g_{N} \xi$. This splitting in turn generates Coleman-Weinberg correction [38] to the tree level potential. The one-loop correction to the potential can be estimated using

$$
\Delta V=\sum_{i} \frac{(-1)^{F} m_{i}^{4}}{64 \pi^{2}} \ln \left(\frac{m_{i}^{2}}{\Lambda^{2}}\right)
$$

where $i$ runs over the scalars and fermions. $\Lambda$ is the ultraviolet cutoff of the theory which we identify with the reduced Planck scale, $M_{P}=1 /(8 \pi G)^{1 / 2}=2.4 \times 10^{18} \mathrm{GeV}$. The oneloop corrected effective potential is then given by

$$
V_{\text {eff }} \equiv V+\Delta V \simeq \frac{1}{2} \xi^{2}\left(1+\frac{g_{N}^{2}}{16 \pi^{2}} \ln \left(\frac{\lambda_{N}^{2} \sigma^{2}}{2 M_{P}^{2}}\right)\right)
$$

for $\lambda_{N}^{2} \sigma^{2} \gg 2 g_{N} \xi$. The constant tree level contribution provides the vacuum energy density required to drive inflation and the slow roll is provided by the one-loop correction.

The values of $g_{N}$ and $\xi$ can be estimated by fitting the potential in Eq. (32) with the observables from inflation models. These observables are minimum number of efoldings $N$, amplitude of temperature anisotropy $A_{s}$, spectral index $n_{s}$, and tensor-scalar ratio $r$. They are given by

$$
\begin{gathered}
A_{s}=\frac{V}{24 \pi^{2} M_{P}^{4} \epsilon}, \\
n_{s}=1-6 \epsilon+2 \eta, \\
N_{\mathrm{CMB}}=\int H_{I} d t=\int_{\sigma_{c}}^{\sigma_{\mathrm{CMB}}} \frac{d \sigma}{M_{P} \sqrt{2 \epsilon}}, \\
r=16 \epsilon .
\end{gathered}
$$

Here, $\epsilon$ and $\eta$ are the slow roll parameters which for the potential in Eq. (32) are obtained as

$$
\begin{aligned}
\epsilon= & \frac{M_{P}^{2}}{2 V_{\mathrm{eff}}^{2}}\left(\frac{\partial V_{\mathrm{eff}}}{\partial \sigma}\right)^{2} \\
= & 2\left(\frac{g_{N}^{2}}{16 \pi^{2}}\right)^{2}\left(\frac{M_{P}}{\sigma}\right)^{2}\left(1+\frac{g_{N}^{2}}{16 \pi^{2}} \ln \left(\frac{\lambda_{N}^{2} \sigma^{2}}{2 M_{P}^{2}}\right)\right)^{-2}, \\
\eta & =\frac{M_{P}^{2}}{V_{\mathrm{eff}}}\left(\frac{\partial^{2} V_{\mathrm{eff}}}{\partial \sigma^{2}}\right) \\
& =-\frac{g_{N}^{2}}{8 \pi^{2}}\left(\frac{M_{P}}{\sigma}\right)^{2}\left(1+\frac{g_{N}^{2}}{16 \pi^{2}} \ln \left(\frac{\lambda_{N}^{2} \sigma^{2}}{2 M_{P}^{2}}\right)\right)^{-1} .
\end{aligned}
$$

$N_{\mathrm{CMB}}$ is the number of e-foldings between the end of inflation and the time when the CMB modes are exiting the inflationary horizon and $\sigma_{\mathrm{CMB}}$ is the value of the inflaton field when the $\mathrm{CMB}$ modes are exiting the inflation horizon. $\sigma_{c}$ is the critical value of $\sigma$ when inflation ends. Inflation can end in two possible ways. If at some value of $\sigma, \epsilon \simeq 1$, then the slow roll phase ends. It is also possible that $\sigma$ reaches the critical value when the local supersymmetry breaking minimum becomes unstable and the fields roll along the $\phi_{N}^{+}$direction. This critical value is given by $\sigma_{c}=\sqrt{2 g_{N} \xi} /\left|\lambda_{N}\right|$.

In the following, we assume $\sigma_{\mathrm{CMB}} \simeq M_{P}$ at the start of inflation so that we get $\mathcal{O}(50)$ e-foldings consistent with the Lyth bound [39]. The different horizons in the Universe can have different values of the inflaton field and the patch of the Universe where $\sigma \gtrsim M_{P}$ will start inflating and will dominate the volume of the Universe. The observables given in Eq. (33) are then functions of model parameters $g_{N}, \lambda_{N}$, and $\xi$. Among these only $A_{s}$ depends on $\xi$. We scan the values of $g_{N}$ and $\lambda_{N}$ to determine all the observables except $A_{s}$. Parameter $\xi$ is then determined using the value of $A_{s}$ as measured by Planck 2018, $A_{s}=(2.099 \pm 0.101) \times$ $10^{-9}$ [40]. The results are displayed in Fig. 3. We find that $n_{s} \leq 0.9775$, the upper $3 \sigma$ limit as measured by Planck 2018 [40], requires $g_{N} \geq 0.92$ almost independent of values of $\lambda_{N}$. For $g_{N}=0.92, N_{\mathrm{CMB}}$ can be as large as 46 for $\lambda_{N} \simeq \sqrt{4 \pi}$. The greater value of $g_{N}$ decreases the number of e-foldings considerably. The scalar to tensor ratio remains well below the upper bound, $r<0.062$. For $g_{N}=0.92$, 

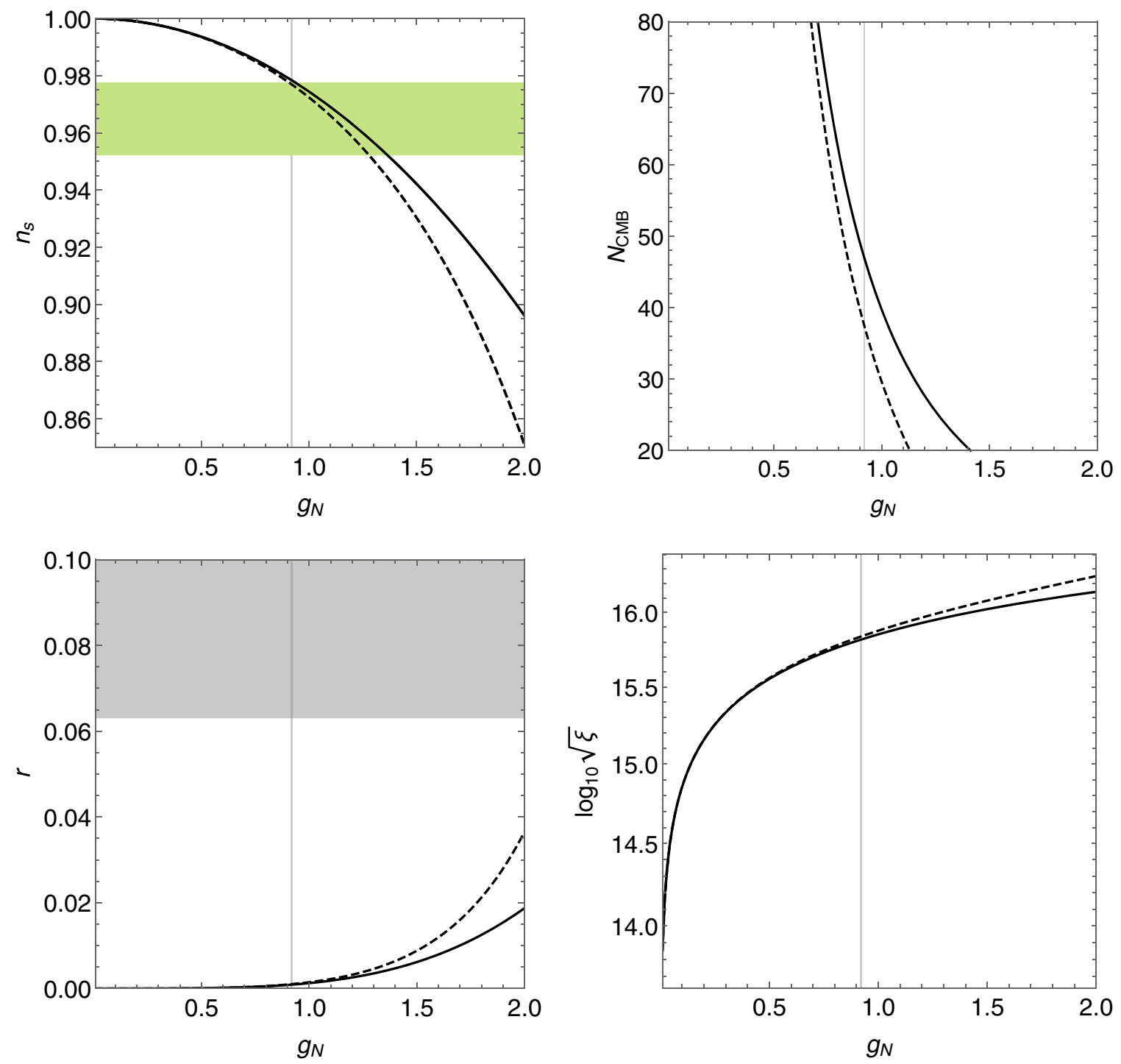

FIG. 3. The spectral index $n_{s}$, number of e-foldings $N_{\mathrm{CMB}}$, scalar to tensor ratio $r$, and the $\xi$ parameter as functions of $g_{N}$ as predicted by the model (see the text for details). The dashed (solid) line corresponds to $\left|\lambda_{N}\right|=10^{-2}\left(\left|\lambda_{N}\right|=\sqrt{4 \pi}\right.$ ). The green band in top-left panel indicates $3 \sigma$ range of $n_{s}=0.9649 \pm 0.0042$ as measured by Planck 2018 [40]. The gray region in the bottom-left panel is excluded by Planck 2018 at $95 \%$ C.L.

the value of $\xi$ fixed from $A_{s}$ is determined as $\sqrt{\xi} \simeq 6.3 \times 10^{15} \mathrm{GeV}$.

The inflation parameters $A_{s}$ and $n_{s}$ measured by Planck 2018 [40] are reported for the scale $k=0.05 \mathrm{Mpc}^{-1}$. The mode with comoving wave number $k$ exits the inflation horizon when the physical length scale of the perturbation $a_{k} / k$ is the size of the horizon $H_{I}^{-1}$, i.e., when $a_{k}=k H_{I}^{-1}$. Therefore, the number of e-foldings $N(k)$ before the end of inflation when a given mode $k$ leaves the inflation horizon given by [41-43]

$e^{N(k)}=\frac{a_{\mathrm{end}}}{a_{k}}=a_{\mathrm{end}} \frac{H_{I}}{k}=\left(\frac{H_{I}}{k}\right)\left(\frac{a_{\mathrm{end}}}{a_{\mathrm{reh}}}\right)\left(\frac{a_{\mathrm{reh}}}{a_{\mathrm{eq}}}\right)\left(\frac{a_{\mathrm{eq}}}{a_{0}}\right)$, which implies

$N(k)=\ln \left(\frac{H_{I}}{k}\right)+\frac{1}{3} \ln \left(\frac{\rho_{\text {reh }}}{\rho_{\text {end }}}\right)+\frac{1}{4} \ln \left(\frac{\rho_{\mathrm{eq}}}{\rho_{\text {reh }}}\right)+\ln \left(\frac{a_{\mathrm{eq}}}{a_{0}}\right)$.

Here, we have assumed that the at the end of inflation the inflaton oscillates at the bottom of the potential and the energy density falls as $\rho \sim a^{-3}$ and then the Universe reheats due to the coupling of inflaton with the SM fields. In Eq. (40), $H_{I}=\left(V / 3 M_{P}^{2}\right)^{1 / 2}$ is the Hubble parameter during inflation where the potential $V \simeq \xi^{2} / 2$. With $\xi=$ $\left(6.3 \times 10^{15} \mathrm{GeV}\right)^{2}$, we obtain the value of $H_{I}=6.65 \times$ $10^{12} \mathrm{GeV}$. The ratio $a_{0} / a_{\mathrm{eq}}=3450$ and $T_{\mathrm{eq}}=0.81 \mathrm{eV}$. 
Using these parameters in Eq. (40) and taking $N(k=$ $\left.0.05 \mathrm{Mpc}^{-1}\right) \simeq 46$, the reheat temperature turns out to be $T_{\text {reh }}=10^{6} \mathrm{GeV}$. Reheating at the end of inflation takes place when the $X$ particles decay into $\phi_{N}^{ \pm}$and gauge bosons.

\section{CONSTRAINTS FROM LONG-RANGE FORCES}

As discussed in the previous section, a viable inflation within this framework requires

$$
g_{N} \simeq 1 \text { and } \sqrt{\xi} \simeq 10^{16} \mathrm{GeV} .
$$

Substitution of the above in Eqs. (25), (27), and (28) determines the allowed values of $g^{\prime}$ and $M_{Z^{\prime}}$ as function of $\mathrm{CW}$ parameters $N, k$, and $q . M_{Z^{\prime}}$ also depends on $v_{\chi} \lesssim v_{k}$. To be more specific, we associate $v_{\chi}$ with the breaking scale of $U(1)_{k}$ by assuming

$$
v_{\chi} \simeq v_{k}=q^{\frac{N-k}{2}} \sqrt{\frac{\xi}{g_{N}}},
$$

where the second equality results from Eq. (10). This, along with the above values of $g_{N}$ and $\xi$, leads to

$$
k=2\left(\frac{\log _{10}\left(M_{Z^{\prime}} / \mathrm{eV}\right)-25}{\log _{10} q}\right)-N
$$

from Eq. (25). Similarly, substitution of the values of $g_{N}, \xi$, and $M_{V_{M}}=1 \mathrm{TeV}$ in Eqs. (27) and (28) implies

$$
N=\frac{\log _{10} g^{\prime}-26}{\log _{10} q} .
$$

Desired value of $g^{\prime}$ and $M_{Z^{\prime}}$ can therefore be obtained by choosing appropriate values of $N$ and $k$ for a given $q$. The ratio $k / N$ however does not depend on the value of $q$ and it can be constrained once the nature of the SM current $J^{\mu}$ is fixed. We do this by identifying $U(1)_{M}$ with $L_{\mu}-L_{\tau}$, $L_{e}-L_{\tau}$, and $B-L$ symmetries.

$$
\text { A. } L_{\mu}-L_{\tau}
$$

We discuss here various constraints which are used to restrict the parameter space in case of $U(1)_{L_{\mu}-L_{\tau}}$ symmetry and its consequences for the $\mathrm{CW}$ setup considered here. The second and the third generation of leptons are charged under $U(1)_{M}$ with charges +1 and -1 , respectively, but the first generation is neutral. Thus, objects containing electrons do not experience the $L_{\mu}-L_{\tau}$ forces and the conventional method used to constrain fifth force do not apply. ${ }^{4}$

\footnotetext{
${ }^{4}$ These constraints become meaningful if $Z^{\prime}$ has a mass mixing $[44,45]$ with the ordinary $B$ boson. We assume that such mixing is not present.
}

But the muon rich astrophysical sources like neutron star binaries can provide significant constrain on ultralight gauge boson. Emission of an ultralight gauge boson of $L_{\mu}-L_{\tau}$ causes a fast decay in the orbital period of a pulsar binary. The observed orbital periods have been used in $[46,47]$ to constrain the mass and couplings of the $L_{\mu}-L_{\tau}$ gauge boson. The masses $M_{Z^{\prime}}$ below around $10^{-10} \mathrm{eV}$ are constrained in this way. This constraint is shown in Fig. 4 as a gray region enclosed by gray line [47].

$M_{Z^{\prime}}$ above $10^{-10} \mathrm{eV}$ but below $m_{\nu_{i}}$ are constrained from the invisible neutrino decays $\nu_{i} \rightarrow \nu_{j}+Z^{\prime}$. The strongest limit on the neutrino lifetime comes from the structure formation in the early Universe [56,57] through CMB observation by Planck 2018 [58]. This constraint is shown in the upper panel of Fig. 4. This limit is however cosmological model dependent and it does not apply if neutrinos disappear before recombination epoch through additional decay channels; see, for example, [59]. A less stringent but more robust bound on invisible neutrino decays come from the laboratory data on nonobservation of the $\nu_{2}$ decays [60] and it disfavors the region shaded by blue in the top panel of Fig. 4. Higher mass range can be constrained from various other considerations. The coupling of the longitudinal $Z^{\prime}$ to neutrinos goes as $g^{\prime} m_{\nu} / M_{Z^{\prime}}$ and could lead to unitarity violation. This provides a limit [48] on $g^{\prime}$ for a larger mass range in $M_{Z^{\prime}}$ as displayed in Fig. 4. Neutrino trident production [50] also provides a strong complementary limit on $g^{\prime}$ for $M_{Z^{\prime}}>m_{\nu}$. This is shown as a region shaded in orange in Fig. 4. As it can be seen, the various constraints still allow $M_{Z^{\prime}} \sim 10^{-14}$ $10^{-18} \mathrm{eV}$ and $g^{\prime} \sim 10^{-26}-10^{-30}$. This region can be obtained in the CW framework for $k=N / 2$ curve displayed in Fig. 4. For example, $N=52, k=26, q=0.1$ give $g^{\prime} \sim 10^{-26}$ and $M_{Z^{\prime}} \sim 10^{-14} \mathrm{eV}$.

The constraints from the invisible neutrino decay and neutrino trident productions used above implicitly assume that the charged lepton mass matrix preserves $L_{\mu}-L_{\tau}$ symmetry and is diagonal. One can consider alternative case with a diagonal neutrino mass matrix. Leptonic mixing then requires a nondiagonal charged lepton mass matrix. In this case, the invisible neutrino decay will be absent at the tree level and constraints from the neutrino trident production would also change. But the charged leptons in this case have flavor violating couplings and $\mu$ and $\tau$ could decay into ultralight $Z^{\prime}$ in this case. We estimate these decays following a similar formalism used in [48] for neutrinos. The $l_{i} \rightarrow l_{j}+Z^{\prime}$ decay width can be obtained as

$$
\begin{aligned}
\Gamma\left[l_{i} \rightarrow l_{j} Z^{\prime}\right]= & \frac{1}{32 \pi m_{l_{i}}^{3}} \frac{g^{\prime 2}}{M_{Z^{\prime}}^{2}}\left|Q_{i j}\right|^{2}\left(m_{l_{i}}^{2}-m_{l_{j}}^{2}\right)^{2} \lambda^{1 / 2} \\
& \times\left(m_{l_{i}}^{2}, m_{l_{j}}^{2}, M_{Z^{\prime}}^{2}\right),
\end{aligned}
$$

where $\lambda(x, y, z)=x^{2}+y^{2}+z^{2}-2 x y-2 y z-2 z x$ and $Q=U_{\text {PMNS }} \hat{Q} U_{\text {PMNS }}^{\dagger}$ with $\hat{Q}=\operatorname{Diag}(0,1,-1)$ for the 

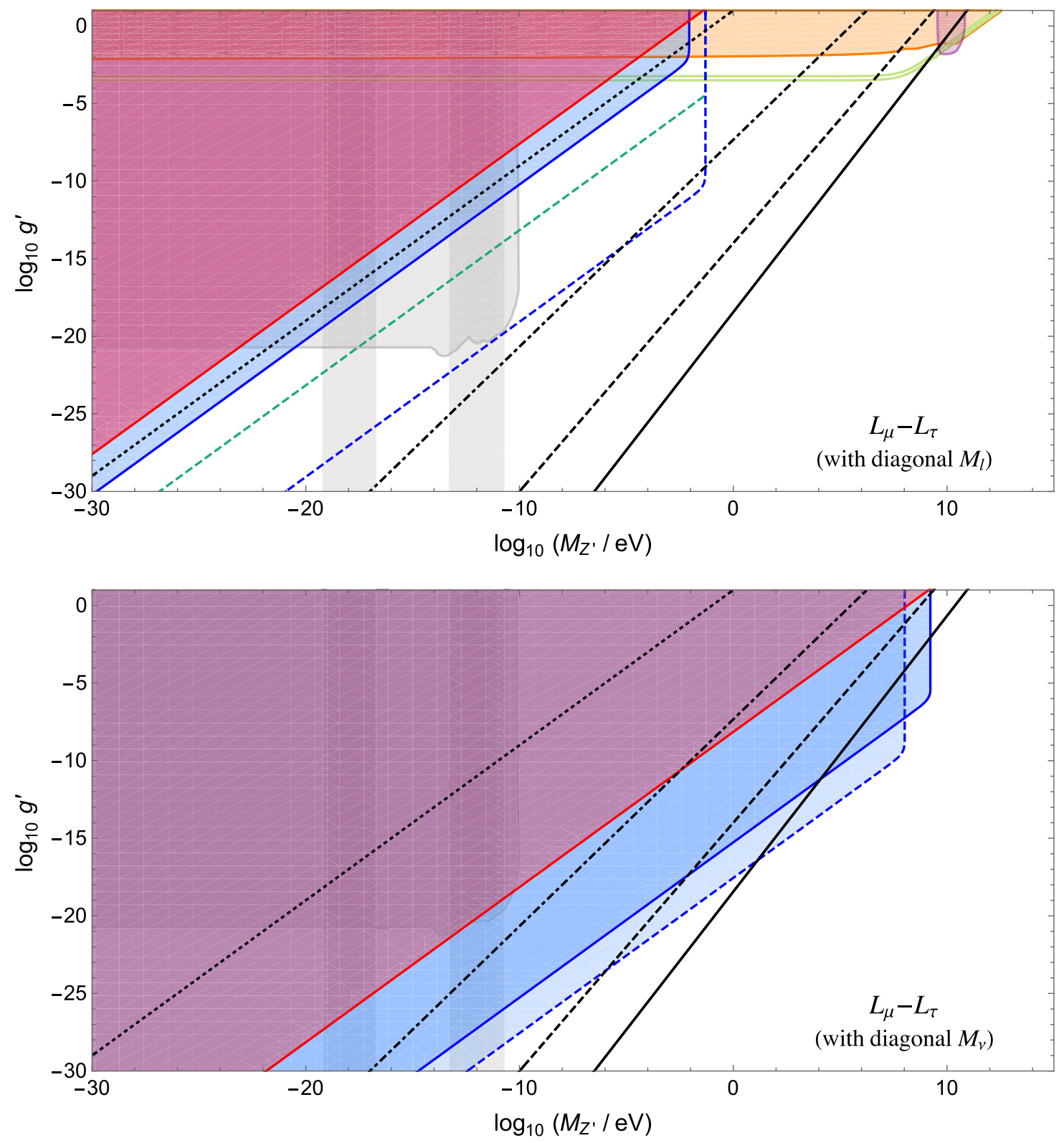

FIG. 4. Constraints on $g^{\prime}$ and $M_{Z^{\prime}}$ for $U(1)_{M}=L_{\mu}-L_{\tau}$ with diagonal $M_{l}\left(M_{\nu}\right)$ in the top (bottom) panel. The black lines represent model predicted correlations for $k / N=1 / 8$ (solid), $1 / 4$ (dashed), $1 / 2$ (dot-dashed), and $k / N=1$ (dotted). The red shaded region is excluded by unitarity limit [48]. The vertical gray bands indicate the range of $M_{Z^{\prime}}$ disfavored by black hole superradiance [49], while the gray region enclosed by gray line is excluded by neutron star binaries [46,47]. Shaded in orange (purple) is the region disfavored by neutrino trident production [50] (LHC [51,52]) constraints. The region enclosed between two green contours is favored by muon $g-2$ at $2 \sigma[1,53]$. Limit set by big bang nucleosynthesis due to neutrino annihilation is shown by dashed green line [48]. The regions enclosed by solid and dashed blue lines in the top panel are disfavored by laboratory and cosmological constraints on neutrino decays [48], respectively. The same in the lower panel are excluded by upper limits on $\operatorname{BR}\left[\tau \rightarrow \mu Z^{\prime}\right]$ [54] and $\mathrm{BR}\left[\mu \rightarrow e Z^{\prime}\right]$ [55], respectively. All the constraints are at $95 \%$ C.L.

underlying case. We use the results of latest fit [61] of neutrino oscillation data to determine the $U_{\mathrm{PMNS}}$ matrix and estimate the branching ratios for the decays, $\tau \rightarrow \mu+Z^{\prime}$ and $\mu \rightarrow e+Z^{\prime}$. The upper limits $\operatorname{BR}\left[\tau \rightarrow \mu Z^{\prime}\right]<5 \times 10^{-3}$ [54] and $\operatorname{BR}\left[\mu \rightarrow e Z^{\prime}\right]<5.8 \times 10^{-5}$ [55] are then used to constrain $g^{\prime}$ and $M_{Z^{\prime}}$ in the lower panel of Fig. 4. As can be seen, these constraints are more powerful and exclude $k / N \gtrsim 1 / 4$ leaving no room for the long-range interactions.

$L_{\mu}-L_{\tau}$ symmetry has also been evoked to explain the current discrepancy between theoretically predicted muon anomalous magnetic moment $(g-2)_{\mu}$ and its experimental 

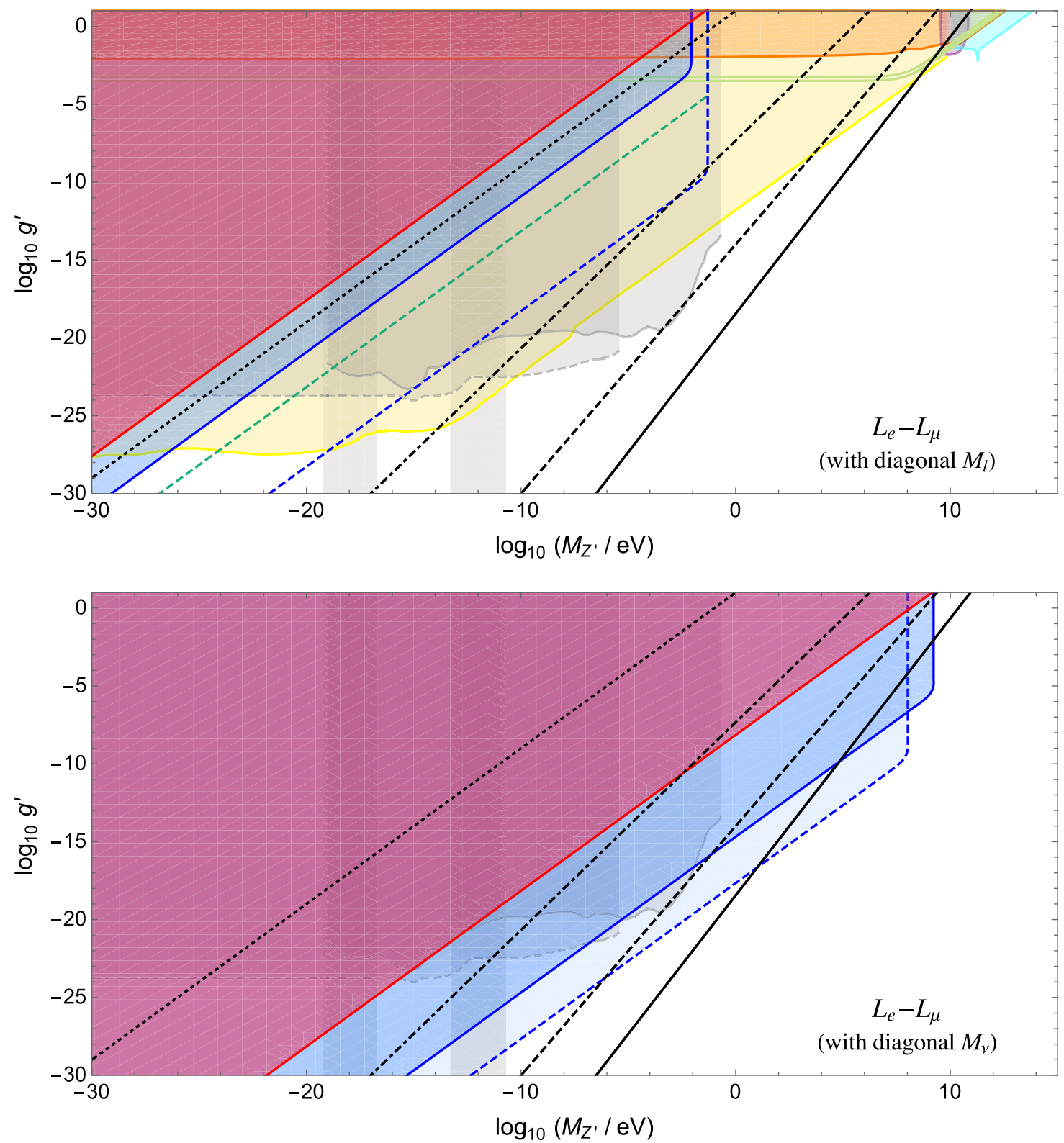

FIG. 5. Constraints on $g^{\prime}$ and $M_{Z^{\prime}}$ for $U(1)_{M}=L_{e}-L_{\mu}$ with diagonal $M_{l}\left(M_{\nu}\right)$ in the top (bottom) panel. The region shaded in yellow is disfavored by matter effects in neutrino oscillation [62,63]. Gray regions enclosed by solid (dashed) gray contours are excluded by experimental test of violation of equivalence principle (fifth force) [62]. The region shaded in cyan is excluded by LEP [64]. All the other details are same as discussed in the caption of Fig. 4.

value. This can be resolved if $g^{\prime} \simeq 10^{-3}$ and $M_{Z^{\prime}} \simeq$ $\mathcal{O}(\mathrm{MeV})$ as seen from the top panel of Fig. 4. Such values can be accommodated in the proposed $\mathrm{CW}$ by taking $N=29, k=9$ for $q=0.1$ as can be seen from Eqs. (43) and (44). For this choice, one obtains $M=3$ for which $M_{V_{M}} \sim \mathrm{TeV}$ as explained earlier.

\section{B. $L_{e}-L_{\mu}$}

In this case, the $Z^{\prime}$ boson has gauge interactions with the first generation leptons and therefore the stringent constraints on $g^{\prime}-M_{Z^{\prime}}$ arise from the matter effects in neutrino oscillations [62] and from the precision tests of gravity. These are more powerful in comparison to the limits from neutrino decays as can be seen from the top panel in Fig. 5 . The constraints still allow some room for the long-range $L_{e}-L_{\mu}$ interaction corresponding to $g^{\prime} \simeq 10^{-27}$ and $M_{Z^{\prime}} \simeq$ $10^{-15} \mathrm{eV}$ which can be incorporated in the proposed $\mathrm{CW}$ setup with $k=27$ and $N=53$ for $q=0.1$.

We also consider the constraints from the charged lepton decays which arise if $L_{e}-L_{\mu}$ is broken by the charged lepton mass matrix and the neutrino mass matrix is diagonal. The same procedure as outlined in the previous 


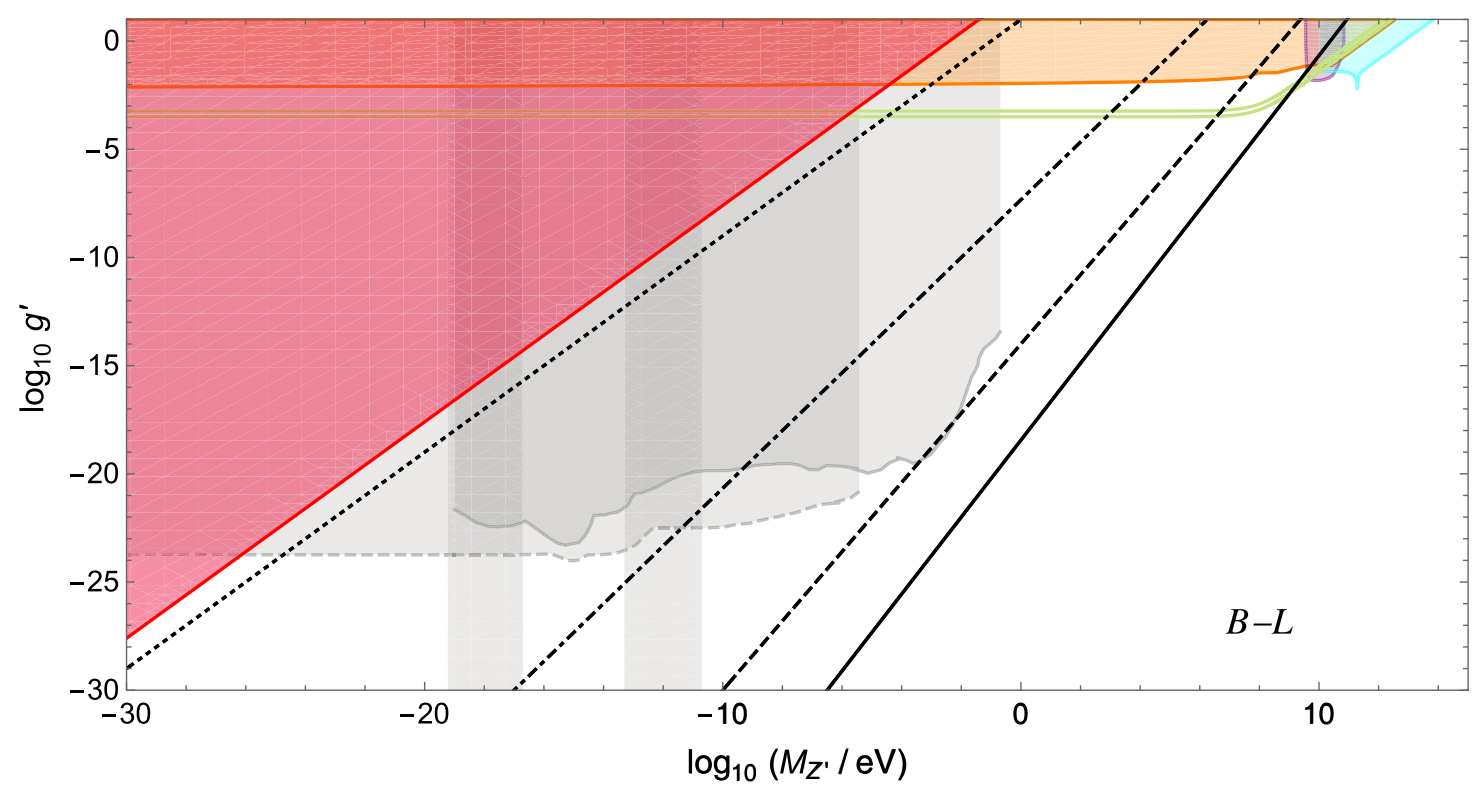

FIG. 6. Constraints on $g^{\prime}$ and $M_{Z^{\prime}}$ for $U(1)_{M}=B-L$. Gray regions enclosed by solid (dashed) gray contours are excluded by experimental test of violation of equivalence principle (fifth force) [62]. The region shaded in cyan is excluded by LEP [64]. All the other details are same as discussed in the caption of Fig. 4. We do not show various other constraints applicable in the range of $M_{Z^{\prime}}$ in 1 eV$10 \mathrm{GeV}$; see $[17,65]$ for their details.

subsection is followed but with $\hat{Q}=\operatorname{Diag} .(1,-1,0)$ in Eq. (45). The flavor violating charged lepton decays provide the most stringent constraints and entirely exclude $k / N \geq 1 / 4$ as displayed in the bottom panel in Fig. 5. Most of the constraints and results discussed in this subsection are also applicable to the $L_{e}-L_{\tau}$ type of interactions.

\section{C. $B-L$}

For $U(1)_{M}=B-L$, the gauge interactions involving $Z^{\prime}$ are flavor universal. The dominant constraint on the ultralight $Z^{\prime}$ comes from the experiments testing the validity of equivalence principle and existence of the fifth force as discussed before. These constrains allow $g^{\prime} \leq 10^{-24}$ and $M_{Z^{\prime}} \simeq 10^{-14}-10^{-16} \mathrm{eV}$ which can be obtained within the proposed CW setup for $N \geq 55$ and $k / N \sim 1 / 2$ as can be seen from Fig. 6. The unitarity constraint shown in red color in Fig. 6 is applicable if neutrinos break the lepton number. Also, we do not show various other constraints applicable in the range of $M_{Z^{\prime}}$ in $1 \mathrm{eV}-10 \mathrm{GeV}$ (see [17,65], for example) which is not of importance in the present context.

\section{SUMMARY}

Extension of the minimal supersymmetric SM by a $U(1)$ gauge group is known to lead to a successful description of inflation through a large FI term $\xi$. We have incorporated this mechanism of the $D$-term inflation into a broader framework containing $N+1$ different $U(1)$ gauge groups coupled with each other through Higgs fields in a clockwork fashion. As discussed here, this offers an exciting possibility of unifying the large scale inflation and long- range interactions mediated by an ultralight gauge boson, and in-turn explains 40 orders of magnitude difference between the scales without relying on any unnatural parameter. Such long-range forces can be minimally incorporated in the SM by gauging $U(1)$ symmetry corresponding to the difference $L_{i}-L_{j}$ of individual lepton number. Such $U(1)$ symmetries form a part of the underlying CW framework. Both tiny mass $\sim 10^{-15} \mathrm{eV}$ of the gauge boson and its extremely weak coupling $g^{\prime} \sim 10^{-26}$ to the SM fields arise from the underlying CW mechanism. Simultaneously, it also allows $\mathcal{O}(1)$ coupling $g_{N}$ needed for $D$-term inflation. As discussed at length in Sec. III, various conditions required for successful inflation can be met within the present scenario.

We have considered in detail three specific cases of the long-range forces generated by the $L_{\mu}-L_{\tau}, L_{e}-L_{\mu}$, and very light $B-L$ gauge bosons. We have collected most of the relevant astrophysical, cosmological, and terrestrial constraints in these scenarios and shown that the values of parameters $g^{\prime}$ and $M_{Z^{\prime}}$ surviving after these constraints can be understood within this framework.

The proposed $\mathrm{CW}$ mechanism is not restricted to the description of the long-range forces. The same setup also allows a heavier $M_{Z^{\prime}}$ (see Fig. 4) or heavier excitations of the lowest mass state (see Fig. 2) with stronger couplings to SM fields than the ones required for the long-range forces. They can be used as an explanation of some other physical situations along with inflation. We considered here a specific case of $M_{Z^{\prime}} \sim \mathcal{O}(\mathrm{MeV})$ with coupling $g^{\prime} \sim 10^{-3}$ that arise in the $\mathrm{CW}$ and provide a possible explanation of the long-standing muon $(g-2)$ discrepancy. 


\section{ACKNOWLEDGMENTS}

We are grateful to Pierre Fayet for pointing out an error in the previous version of the paper. The work of K. M. P. was partially supported by research grant under INSPIRE Faculty Award (DST/INSPIRE/04/2015/000508) from the Department of Science and Technology, Government of India.

[1] S. Baek, N. G. Deshpande, X. G. He, and P. Ko, Muon anomalous $g-2$ and $L_{\mu}-L_{\tau}$ models, Phys. Rev. D 64, 055006 (2001).

[2] M. Lindner, M. Platscher, and F. S. Queiroz, A call for new physics: The muon anomalous magnetic moment and lepton flavor violation, Phys. Rep. 731, 1 (2018).

[3] W. Altmannshofer, S. Gori, S. Profumo, and F. S. Queiroz, Explaining dark matter and B decay anomalies with an $L_{\mu}-L_{\tau}$ model, J. High Energy Phys. 12 (2016) 106.

[4] B. Holdom, Two U(1)'s and Epsilon charge shifts, Phys. Lett. B 166, 196 (1986).

[5] D. Hooper, N. Weiner, and W. Xue, Dark forces and light dark matter, Phys. Rev. D 86, 056009 (2012).

[6] M. Fabbrichesi, E. Gabrielli, and G. Lanfranchi, The dark photon, arXiv:2005.01515.

[7] S. Hannestad, R. S. Hansen, and T. Tram, How SelfInteractions Can Reconcile Sterile Neutrinos with Cosmology, Phys. Rev. Lett. 112, 031802 (2014).

[8] B. Dasgupta and J. Kopp, Cosmologically Safe eV-Scale Sterile Neutrinos and Improved Dark Matter Structure, Phys. Rev. Lett. 112, 031803 (2014).

[9] E. D. Stewart, Inflation, supergravity and superstrings, Phys. Rev. D 51, 6847 (1995).

[10] P. Binetruy and G. R. Dvali, D term inflation, Phys. Lett. B 388, 241 (1996).

[11] E. Halyo, Hybrid inflation from supergravity D terms, Phys. Lett. B 387, 43 (1996).

[12] P. Fayet, A new long range force?, Phys. Lett. B 171, 261 (1986).

[13] P. Fayet, The fifth force charge as a linear combination of baryonic, leptonic (Or $B^{-}$l) and electric charges, Phys. Lett. B 227, 127 (1989).

[14] X. G. He, G. C. Joshi, H. Lew, and R. R. Volkas, New Z-prime phenomenology, Phys. Rev. D 43, R22 (1991).

[15] X.-G. He, G. C. Joshi, H. Lew, and R. R. Volkas, Simplest Z-prime model, Phys. Rev. D 44, 2118 (1991).

[16] R. Foot, X. G. He, H. Lew, and R. R. Volkas, Model for a light Z-prime boson, Phys. Rev. D 50, 4571 (1994).

[17] J. Heeck, Unbroken B-L symmetry, Phys. Lett. B 739, 256 (2014).

[18] P. Fayet and J. Iliopoulos, Spontaneously broken supergauge symmetries and Goldstone spinors, Phys. Lett. B 51, 461 (1974).

[19] P. Touboul et al., Microscope Mission: First Results of a Space Test of the Equivalence Principle, Phys. Rev. Lett. 119, 231101 (2017).

[20] D. J. Kapner, T. S. Cook, E. G. Adelberger, J. H. Gundlach, B. R. Heckel, C. D. Hoyle, and H. E. Swanson, Tests of the
Gravitational Inverse-Square Law Below the Dark-Energy Length Scale, Phys. Rev. Lett. 98, 021101 (2007).

[21] S. Schlamminger, K.-Y. Choi, T. A. Wagner, J. H. Gundlach, and E. G. Adelberger, Test of the Equivalence Principle Using a Rotating Torsion Balance, Phys. Rev. Lett. 100, 041101 (2008).

[22] A. S. Joshipura and S. Mohanty, Constraints on flavor dependent long range forces from atmospheric neutrino observations at super-Kamiokande, Phys. Lett. B 584, 103 (2004).

[23] J. A. Grifols and E. Masso, Neutrino oscillations in the sun probe long range leptonic forces, Phys. Lett. B 579, 123 (2004).

[24] A. Yu. Smirnov and X.-J. Xu, Wolfenstein potentials for neutrinos induced by ultra-light mediators, J. High Energy Phys. 12 (2019) 046.

[25] P. Fayet, Spontaneously broken supersymmetric GUTs with gauge Boson/Higgs boson unification, Phys. Lett. B 142, 263 (1984).

[26] P. Fayet, Extra U(1)'s and new forces, Nucl. Phys. B347, 743 (1990).

[27] P. Fayet, The light $U$ boson as the mediator of a new force, coupled to a combination of $Q, B, L$ and dark matter, Eur. Phys. J. C 77, 53 (2017).

[28] P. Fayet, Microscope limits for new long-range forces and implications for unified theories, Phys. Rev. D 97, 055039 (2018).

[29] P. Fayet, Microscope limits on the strength of a new force, with comparisons to gravity and electromagnetism, Phys. Rev. D 99, 055043 (2019).

[30] D. E. Kaplan and R. Rattazzi, Large field excursions and approximate discrete symmetries from a clockwork axion, Phys. Rev. D 93, 085007 (2016).

[31] G. F. Giudice and M. McCullough, A clockwork theory, J. High Energy Phys. 02 (2017) 036.

[32] H. M. Lee, Gauged $U(1)$ clockwork theory, Phys. Lett. B 778, 79 (2018).

[33] A. Ahmed and B. M. Dillon, Clockwork Goldstone bosons, Phys. Rev. D 96, 115031 (2017).

[34] W. Fischler, H. P. Nilles, J. Polchinski, S. Raby, and L. Susskind, Vanishing Renormalization of the D Term in Supersymmetric U(1) Theories, Phys. Rev. Lett. 47, 757 (1981).

[35] J. L. Evans, T. Gherghetta, N. Nagata, and M. Peloso, Lowscale D-term inflation and the relaxion mechanism, Phys. Rev. D 95, 115027 (2017).

[36] V. Domcke and K. Schmitz, Unified model of D-term inflation, Phys. Rev. D 95, 075020 (2017). 
[37] V. Domcke and K. Schmitz, Inflation from high-scale supersymmetry breaking, Phys. Rev. D 97, 115025 (2018).

[38] S. R. Coleman and E. J. Weinberg, Radiative corrections as the origin of spontaneous symmetry breaking, Phys. Rev. D 7, 1888 (1973).

[39] D. H. Lyth, What Would We Learn by Detecting a Gravitational Wave Signal in the Cosmic Microwave Background Anisotropy?, Phys. Rev. Lett. 78, 1861 (1997).

[40] Y. Akrami et al. (Planck Collaboration), Planck 2018 results. X. Constraints on inflation, Astrophys. Space Sci. 641, A10 (2019).

[41] A. R. Liddle and D. H. Lyth, The cold dark matter density perturbation, Phys. Rep. 231, 1 (1993).

[42] A. R. Liddle and S. M. Leach, How long before the end of inflation were observable perturbations produced?, Phys. Rev. D 68, 103503 (2003).

[43] S. Dodelson and L. Hui, A Horizon Ratio Bound for Inflationary Fluctuations, Phys. Rev. Lett. 91, 131301 (2003).

[44] H. Davoudiasl, H.-S. Lee, and W. J. Marciano, Long-range lepton flavor interactions and neutrino oscillations, Phys. Rev. D 84, 013009 (2011).

[45] J. Heeck and W. Rodejohann, Gauged $L_{\mu}-L_{\tau}$ and different muon neutrino and anti-neutrino oscillations: MINOS and beyond, J. Phys. G 38, 085005 (2011).

[46] T. K. Poddar, S. Mohanty, and S. Jana, Vector gauge boson radiation from compact binary systems in a gauged $L_{\mu}-L_{\tau}$ scenario, Phys. Rev. D 100, 123023 (2019).

[47] J. A. Dror, R. Laha, and T. Opferkuch, Probing muonic forces with neutron star binaries, Phys. Rev. D 102, 023005 (2020).

[48] J. A. Dror, Discovering leptonic forces using nonconserved currents, Phys. Rev. D 101, 095013 (2020).

[49] M. Baryakhtar, R. Lasenby, and M. Teo, Black hole superradiance signatures of ultralight vectors, Phys. Rev. D 96, 035019 (2017).

[50] W. Altmannshofer, S. Gori, M. Pospelov, and I. Yavin, Neutrino Trident Production: A Powerful Probe of New Physics with Neutrino Beams, Phys. Rev. Lett. 113, 091801 (2014).

[51] G. Aad et al. (ATLAS Collaboration), Measurements of Four-Lepton Production at the Z Resonance in pp Collisions at $\sqrt{s}=7$ and $8 \mathrm{TeV}$ with ATLAS, Phys. Rev. Lett. 112, 231806 (2014).

[52] S. Chatrchyan et al. (CMS Collaboration), Observation of $\mathrm{Z}$ decays to four leptons with the CMS detector at the LHC, J. High Energy Phys. 12 (2012) 034.

[53] T. Aoyama et al., The anomalous magnetic moment of the muon in the standard model, Phys. Rep. 887, 1 (2020).

[54] H. Albrecht et al. (ARGUS Collaboration), A search for lepton flavor violating decays $\tau \rightarrow \mathrm{e} \alpha, \tau \rightarrow \mu \alpha$, Z. Phys. C 68, 25 (1995).

[55] R. Bayes et al. (TWIST Collaboration), Search for two body muon decay signals, Phys. Rev. D 91, 052020 (2015).

[56] S. Hannestad, Structure formation with strongly interacting neutrinos-Implications for the cosmological neutrino mass bound, J. Cosmol. Astropart. Phys. 02 (2005) 011.

[57] S. Hannestad and G. Raffelt, Constraining invisible neutrino decays with the cosmic microwave background, Phys. Rev. D 72, 103514 (2005).

[58] M. Escudero and M. Fairbairn, Cosmological constraints on invisible neutrino decays revisited, Phys. Rev. D 100, 103531 (2019).

[59] J. F. Beacom, N. F. Bell, and S. Dodelson, Neutrinoless Universe, Phys. Rev. Lett. 93, 121302 (2004).

[60] B. Aharmim et al. (SNO Collaboration), Constraints on neutrino lifetime from the Sudbury Neutrino Observatory, Phys. Rev. D 99, 032013 (2019).

[61] I. Esteban, M. C. Gonzalez-Garcia, M. Maltoni, T. Schwetz, and A. Zhou, The fate of hints: Updated global analysis of three-flavor neutrino oscillations, J. High Energy Phys. 09 (2020) 178.

[62] M. B. Wise and Y. Zhang, Lepton flavorful fifth force and depth-dependent neutrino matter interactions, J. High Energy Phys. 06 (2018) 053.

[63] M. Bustamante and S. K. Agarwalla, Universe's Worth of Electrons to Probe Long-Range Interactions of High-Energy Astrophysical Neutrinos, Phys. Rev. Lett. 122, 061103 (2019).

[64] S. Schael et al. (ALEPH, DELPHI, L3, OPAL, and LEP Electroweak Collaborations), Electroweak measurements in electron-positron collisions at W-boson-pair energies at LEP, Phys. Rep. 532, 119 (2013).

[65] P. Ilten, Y. Soreq, M. Williams, and W. Xue, Serendipity in dark photon searches, J. High Energy Phys. 06 (2018) 004. 